\title{
Editorial
}

\section{Nanomaterials for Optical Sensing and Sensors: Plasmonics, Raman, and Optofluidics}

\author{
Zhida Xu, ${ }^{1}$ Meng Lu, ${ }^{2}$ Hyunjong Jin, ${ }^{3}$ Tao Chen, ${ }^{4}$ and Tiziana C. Bond ${ }^{5}$ \\ ${ }^{1}$ University of Illinois at Urbana-Champaign, Urbana, IL 61801, USA \\ ${ }^{2}$ Iowa State University, Ames, IA 50011, USA \\ ${ }^{3}$ Samsung Electronics, Hwaseong, Gyeonggi, Republic of Korea \\ ${ }^{4}$ Shanghai Institute of Technical Physics, Chinese Academy of Sciences, Shanghai 200083, China \\ ${ }^{5}$ Lawrence Livermore National Laboratory, Livermore, CA 94550, USA \\ Correspondence should be addressed to Zhida Xu; zhidaxul@illinois.edu
}

Received 6 August 2015; Accepted 13 August 2015

Copyright (C) 2015 Zhida Xu et al. This is an open access article distributed under the Creative Commons Attribution License, which permits unrestricted use, distribution, and reproduction in any medium, provided the original work is properly cited.

To date, environmental and healthcare problems have become the most important and challenging issues globally. Demands for fast and low-cost sensing methods and devices for environment monitoring [1], point-of-care diagnosis [2], and food and water safety control [3] are growing rapidly over the years, especially in the underdeveloped areas. Thanks to the advancement of nanotechnology in the past decade, nanomaterials, especially when combined with modern optical sensing techniques, provide us with many new tools, such as surface-enhanced Raman spectroscopy [4], surface plasmon resonance [5], photonic crystal [6], and optofluidics [7], for the sensing applications not accessible by traditional sensing techniques. In the recent decade the research in this field has become flourishing. Figure 1 shows the number of publications in this field according to Thomson Reuters' Web of Science. From Figure 1 we can see that the number of scientific publications has been sky-rocketing. In order to cover the recent progress and ongoing work in this booming field, this timely special issue is dedicated to synthesis, fabrication, characterization, and numerical modeling of nanomaterials and nanostructures with potential applications in biomedical researches [8], environment monitoring [9, 10], explosives trace detection [11], and so on.

This special issue contains 6 articles which cover the academic fields of environmental science, materials science, life science, nanotechnology, optics, physics, and chemistry.
In the research article "Investigation of the Validity of the Universal Scaling Law on Linear Chains of Silver Nanoparticles," M. Alsawafta et al. examined (the generalization of) the universal scaling behavior for spherical silver nanoparticles arranged in finite linear chains (eight nanoparticles) and embedded in different host media. The results of simulation show that the plasmon ruler equation can be successfully extended to represent the fractional plasmon shift of many interacting nanoparticles. The decay length and the mount of the fractional shift of the silver linear chains strongly depend on the polarization state of the incident polarization. The LM exhibits a significant shift of the plasmon resonance (indicated by the value of $k$ ) as compared to that of the TM. This can be explained by the fact that the enhancement of the local field under parallel polarization is more pronounced. On the other hand, the decay of the TM shows a strong dependency on the dielectric function of the surrounding medium. It decays twice faster than the LM, as the host medium becomes denser.

In the research article "Focused Ion Beam Assisted Interface Detection for Fabricating Functional Plasmonic Nanostructures," H. Wang et al. proposed the FIB-assisted interface detection and successfully carried out it using the sampleabsorbed current as the detection signal, and the patterning depth control for the plasmonic structure fabrication was achieved through controlling machining time or ion dose using the EPM. Material-dependent currents were measured 


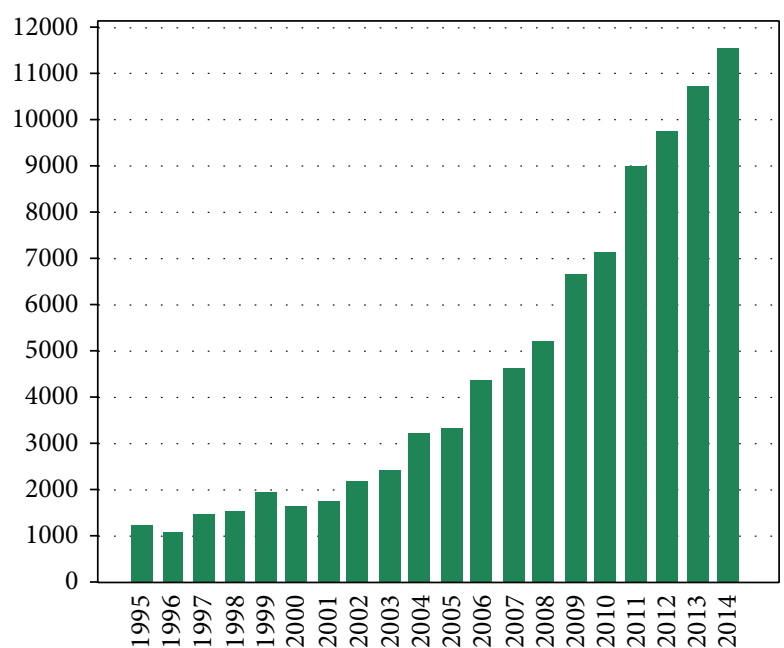

FIGURE 1: Number of publications in the field of optical sensing based on plasmonics and Raman spectroscopy for each year from 1995 to 2014. Source from Thomson Reuters' Web of Science.

through the EPM signal curves, and the quantitative models for the sample-absorbed currents and the ion beam current were also developed.

In the research article "Immobilization Techniques and Integrated Signal Enhancement for POC Nanocolor Microfluidic Devices," M. Schlauf et al. reported the development of a silver enhancement technology that operates even in the presence of high chloride concentrations as it may be encountered in biologic samples. The silver enhancement reagents may be integrated into the microfluidic assay platform to be released upon sample addition. Hereby a highly sensitive onestep-assay can be realized. M. Schlauf et al. also demonstrated that adsorptive immobilization via a cationic polymeric interlayer is a competitive and fast technique for the binding of the capture protein streptavidin onto planar $\mathrm{SiO}_{2}$-surfaces such as REA biochips.

In the research article "Investigating the Fabrication Imperfections of Plasmonic Nanohole Arrays and its Effect on the Optical Transmission Spectra," A. M. Mahros et al. investigated the extraordinary optical transmission spectra of thin gold films perforated with imperfect nano hole arrays using the finite difference time domain (FDTD) method. Exponential shapes for the nanohole sidewalls are used. The investigation of transmission spectra of imperfect nanohole arrays has not previously been demonstrated. It was found that the asymmetry between the two openings of the circular nanoholes or bending to their sidewalls strongly modifies both of the intensity and resonance positions of the transmission spectra. Furthermore, the results of this study assist in explaining the technicality of extraordinary optical transmission phenomenon and why some experimental results on transmission differ from those expected.

In the research article "FRET-Based Detection of Enzymatic Reaction of Botulinum on Microfluidic Device," Y. M. Bae et al. implemented a microfluidic device to detect the enzymatic reaction of botulinum toxin A (BTA) using Förster resonance energy transfer (FRET). The microfluidic device comprised a main channel having two loading zones, a reaction chamber, and a side channel perpendicular to the main channel. The reaction chamber defined by weir in the main channel was packed with microbeads. The movement of the peptide substrate and the BTA in the microfluidic device was controlled by electrophoresis, and the enzymatic reaction of the BTA was detected through the changes of the fluorescence intensity in the reaction chamber. As a result, it was observed that the enzymatic reaction was affected by the electric voltage applied for the movement of the BTA and the peptide and improved by packing the microbeads in the reaction chamber. The microfluidic device provides with the tool to investigate the proteolysis of the substrate by the BTA.

In the research article "Molecular Logic Computation with Debugging Method," X. Liu et al. considered constructing full adder and serial binary adder, using the new concept of seesaw gate. The simulation of the full add preformed properly as designed; however, unexpected exception is noted in the simulation of the serial binary adder. To identify and address the exception, they propose a new method for debugging the molecular circuit. The main idea for this method is to add fan-outs to monitor the circuit in a reverse stepwise manner. These fan-outs are fluorescent signals that can obtain the real-time concentration of the target molecule. By analyzing the monitoring result, the exception can be identified and located. In this paper, examples of XOR and serial binary adder circuits are described to prove the practicability and validity of the molecular circuit debugging method.

\section{Acknowledgments}

The guest editorial team would like to thank the authors for their contributions to this special issue and the reviewers for their time and dedication.

\section{Zhida Xu Meng $L u$ Hyunjong Jin Tao Chen Tiziana C. Bond}

\section{References}

[1] C. K. Ho, A. Robinson, D. R. Miller, and M. J. Davis, "Overview of sensors and needs for environmental monitoring," Sensors, vol. 5, no. 1-2, pp. 4-37, 2005.

[2] R. Kumar, G. P. Singh, I. Barman, N. C. Dingari, and G. Nabi, "A facile and real-time spectroscopic method for biofluid analysis in point-of-care diagnostics," Bioanalysis, vol. 5, no. 15, pp. 18531861, 2013.

[3] R. H. Farahi, A. Passian, L. Tetard, and T. Thundat, "Critical issues in sensor science to aid food and water safety," ACS Nano, vol. 6, no. 6, pp. 4548-4556, 2012.

[4] Z. Xu, J. Jiang, M. R. Gartia, and G. L. Liu, "Monolithic integrations of slanted silicon nanostructures on 3D microstructures and their application to surface-enhanced raman spectroscopy," Journal of Physical Chemistry C, vol. 116, no. 45, pp. 24161-24170, 2012. 
[5] A. G. Brolo, "Plasmonics for future biosensors," Nature Photonics, vol. 6, no. 11, pp. 709-713, 2012.

[6] J. Li, M. D. Muntasir Hossain, B. Jia, D. Buso, and M. Gu, "Threedimensional hybrid photonic crystals merged with localized plasmon resonances," Optics Express, vol. 18, no. 5, pp. 44914498, 2010.

[7] Z. Xu, K. Han, I. Khan, X. Wang, and G. L. Liu, "Liquid refractive index sensing independent of opacity using an optofluidic diffraction sensor," Optics Letters, vol. 39, no. 20, pp. 6082-6085, 2014.

[8] D. J. Bornhop, J. C. Latham, A. Kussrow, D. A. Markov, R. D. Jones, and H. S. Sørensen, "Free-solution, label-free molecular interactions studied by back-scattering interferometry," Science, vol. 317, no. 5845, pp. 1732-1736, 2007.

[9] Z. Xu, Y. Chen, M. R. Gartia, J. Jiang, and G. L. Liu, "Surface plasmon enhanced broadband spectrophotometry on black silver substrates," Applied Physics Letters, vol. 98, no. 24, Article ID 241904, 2011.

[10] W. Shotyk and M. Krachler, "Contamination of bottled waters with antimony leaching from polyethylene terephthalate (PET) increases upon storage," Environmental Science and Technology, vol. 41, no. 5, pp. 1560-1563, 2007.

[11] A. Chou, E. Jaatinen, R. Buividas et al., "SERS substrate for detection of explosives," Nanoscale, vol. 4, no. 23, pp. 7419-7424, 2012. 

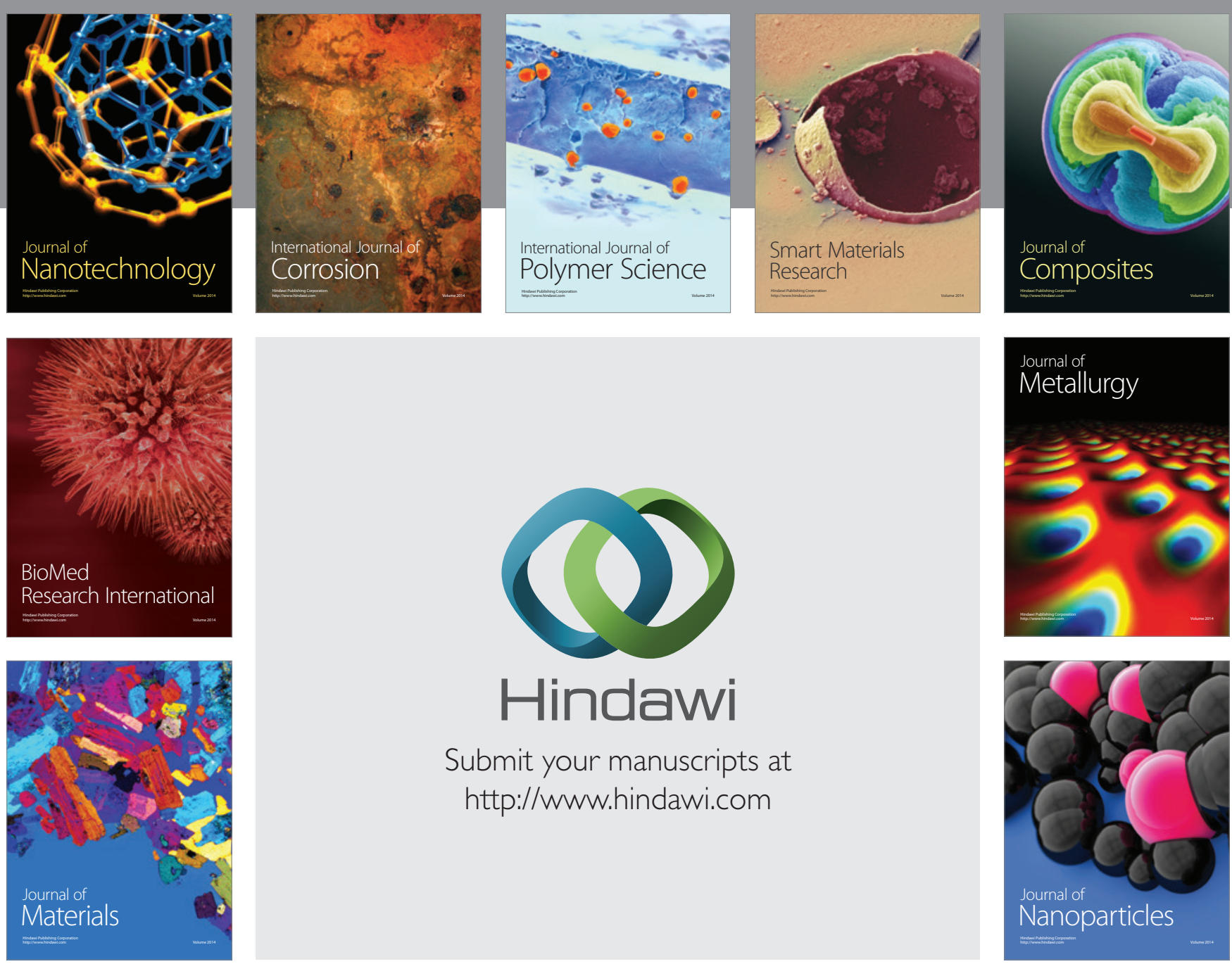

Submit your manuscripts at http://www.hindawi.com
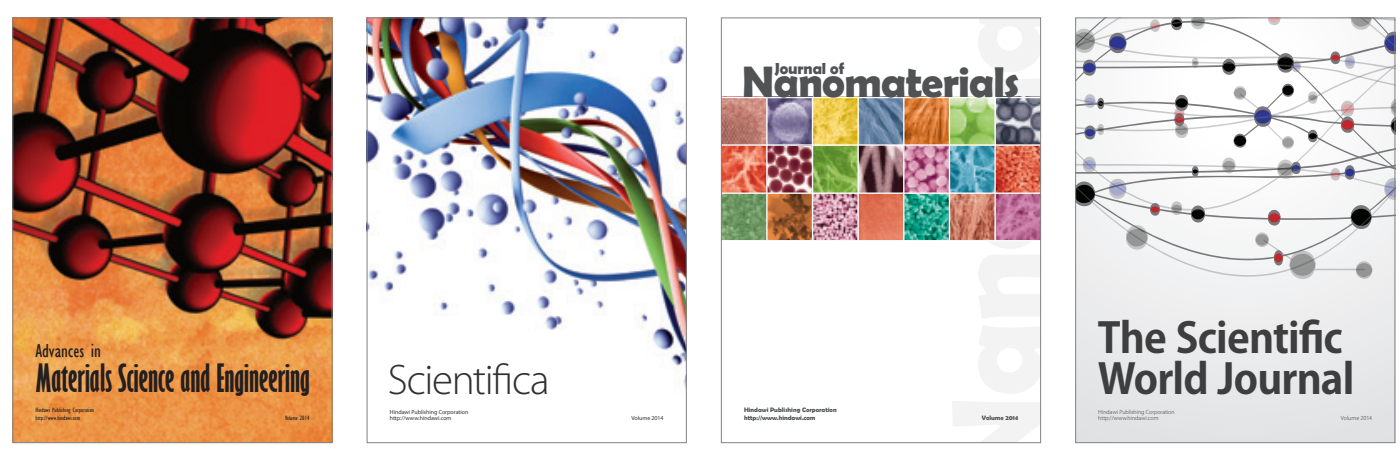

\section{The Scientific World Journal}
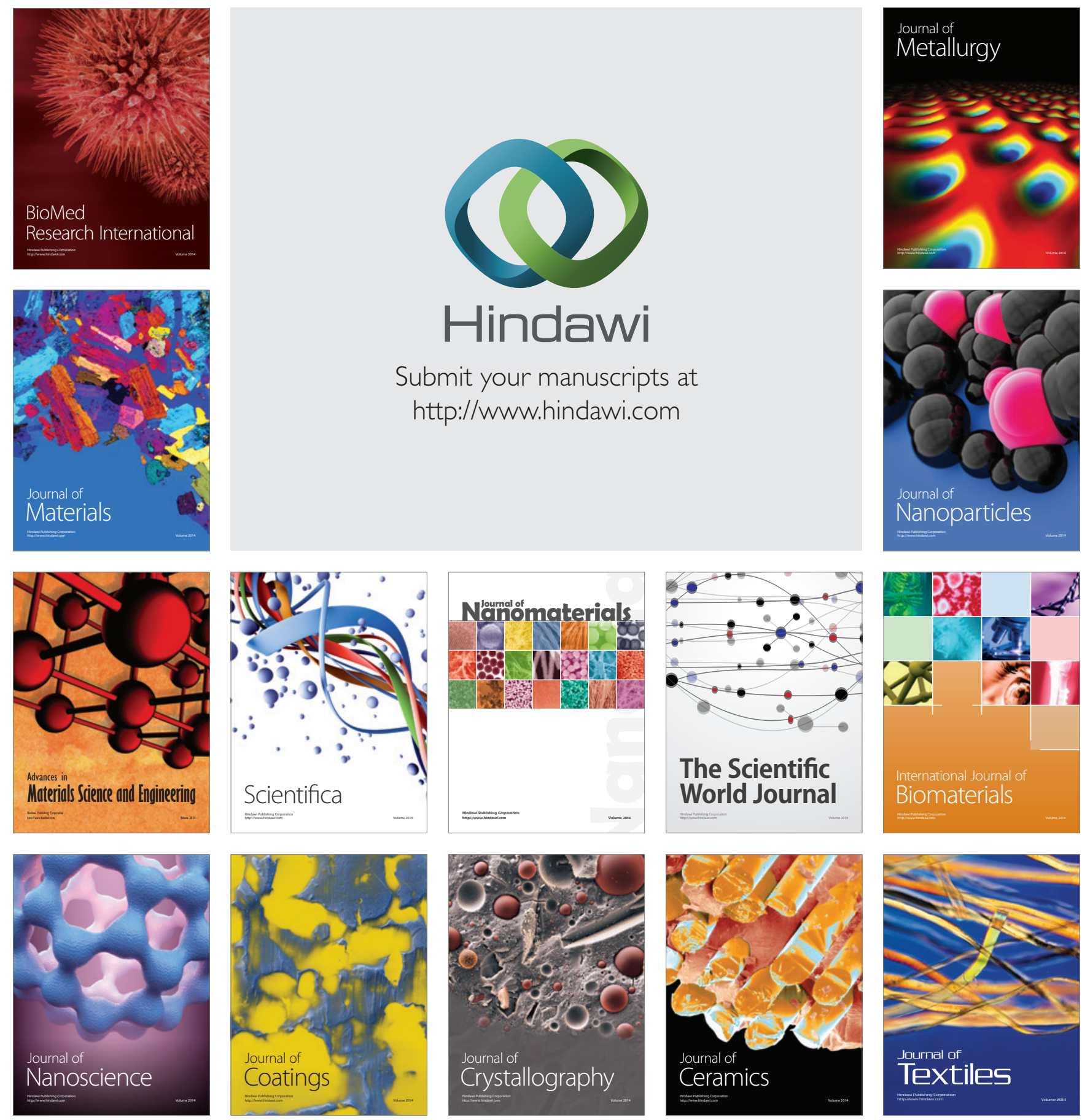